\title{
Development of the Ruthenium Marking System for Local Defect Observation of Zeolite Membrane
}

Kouhei Takatani, Kiyofumi mori, takashi Okabe and Hajime Matsumoto

Mitsubishi Chemical Corporation, Yokohama, Kanagawa, Japan

Zeolite membranes grown on porous alumina have attracted attention as a gas-separating material with little loss. And its separation performance is known to have a large effect on the presence of slight defects. The defects are at gas leaking site and believed some scale such as a cluck and threading lattice defect. Needless to say, it is important to know the location, effect, and size of the defects in nano-scale. In previous work, we had been classed defects as follows;

(1)By submerging the zeolite membrane in the liquid and sending gas from the inside, it is possible to confirm the locations where bubbles leak to the zeolite membrane surface.

(2)The location can be selected with a pen mark in the scanning electron microscope.

(3)If a specific crack or abnormal growth of crystal causes gas leakage, it can be easily found using a scanning electron microscope. However, if it is caused by nanoscale defects, it is difficult to find the location.

In this work, some zeolite membranes containing defects outside the synthesis conditions was used as sample. As preparation for direct defect observation, we have developed a system that combines a local gas detection with a marking technology for FIB-SEM. The location and effects of the defects were investigated using developed by improving the method proposed by Funke et al. [1], and the gas permeated by scanning the tube connected to the mass spectrometer (Figure 1 mode A). It was measured as a quantity. In order to determine the penetrating defect using microscope, marking was performed using ruthenium tetroxide. Ruthenium tetroxide was sealed inside the zeolite tube installed in the above system, and was heated by vacuum evacuation on the outside to selectively adsorb to penetration defects (Figure 1 mode B). Used microscope were Helios Nano Lab 600i dual beam (FEI Company) and TECNAI G2 transmission electron microscopy (FEI Company).

The defect position with ruthenium element was visible in SE image or EDS Ru-map (Figure 2). Using the ruthenium marking system, the Dual-Beam and STEM technique, the defect in a zeolite membrane had been observed from micro-scale to atomic-scale. We had achieved that the cause of the defect generation could be estimated. 
Modo A
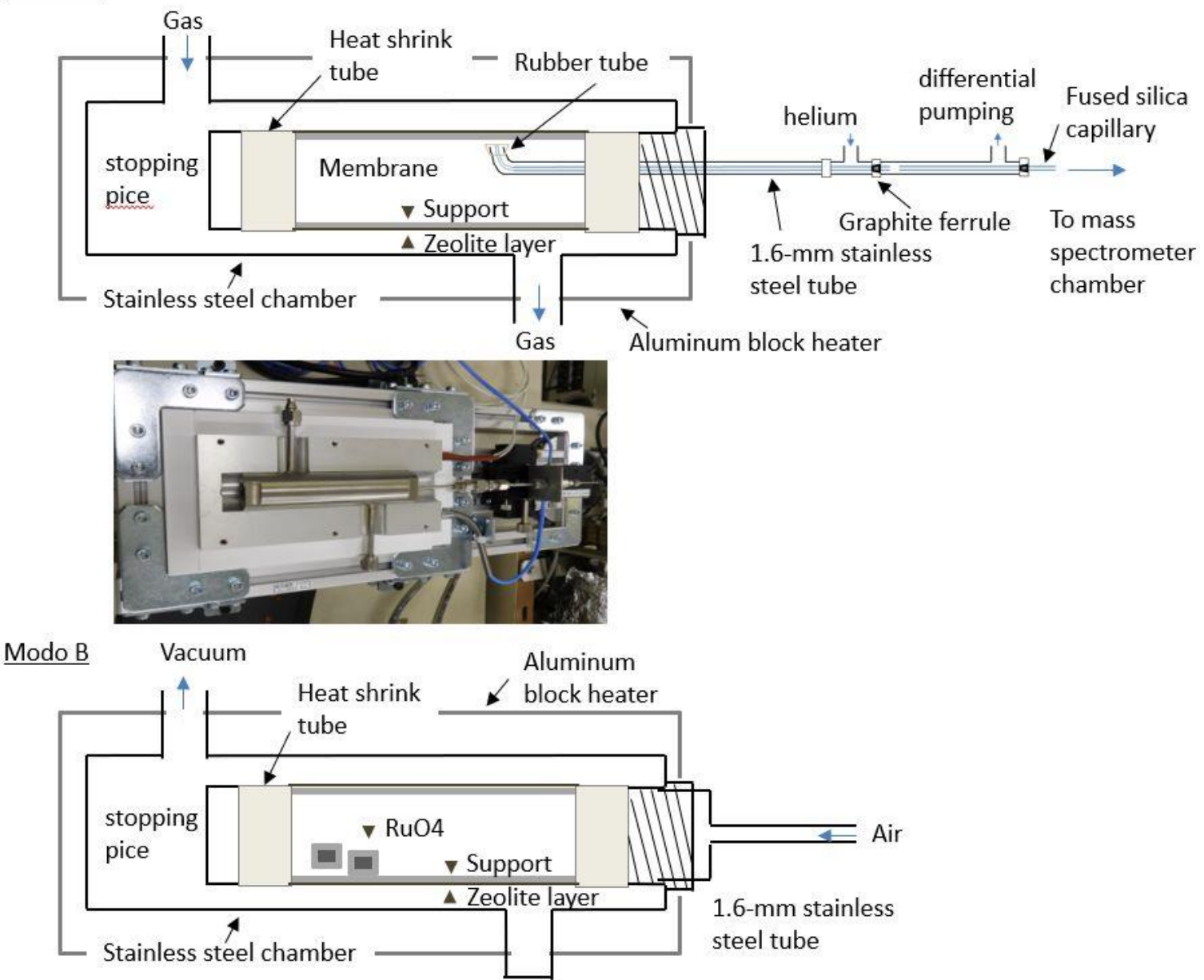

Figure 1. Schematic of local area gas detection system (mode A), and ruthenium marking system (mode B) of zeolite membranes.
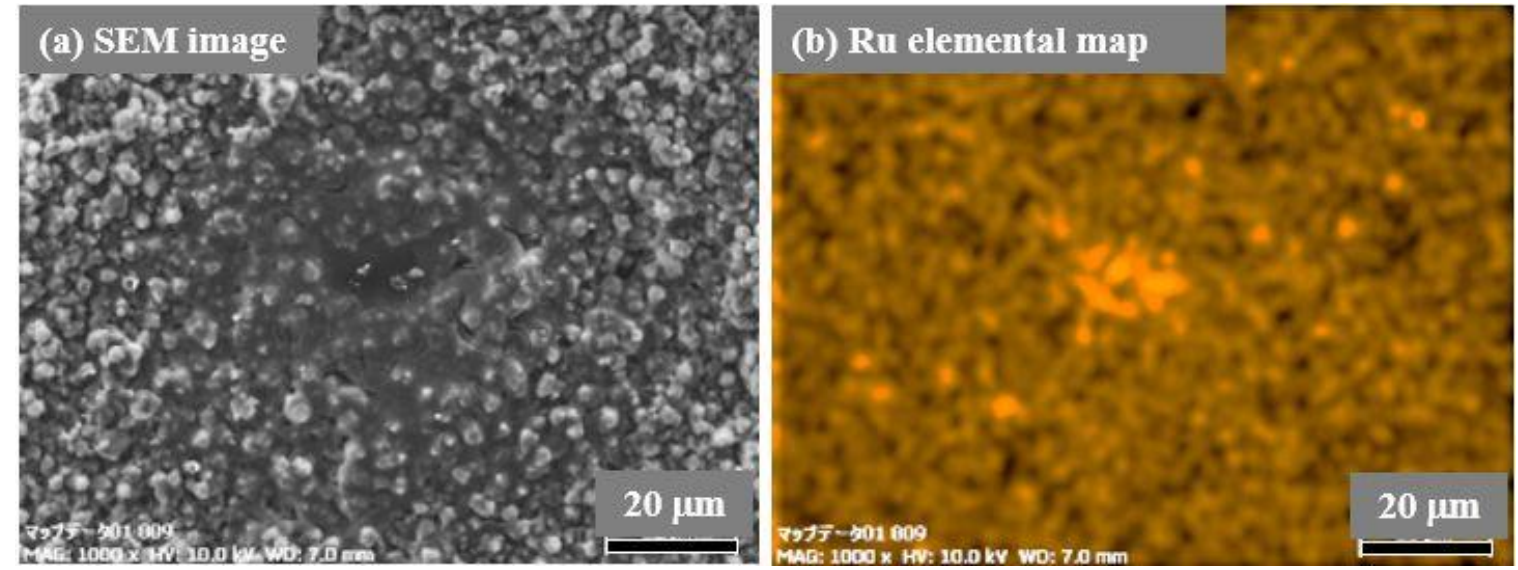

Figure 2. (a) SEM image and (b) $\mathrm{Ru}$ elementale map at the same area of (a) of a zeolite membrane reacted Ruthenium tetroxide.

\section{References}

[1] H. H. Funkeet al., Journal of Membrane Science409-410, 212-221. (2012). 